\title{
Innermost stable circular orbits around relativistic rotating stars
}

\author{
Masaru Shibata and Misao Sasaki \\ Department of Earth and Space Science, Graduate School of Science, Osaka University, \\ Toyonaka, Osaka 560-0043, Japan
}

\begin{abstract}
We investigate the innermost stable circular orbit (ISCO) of a test particle moving on the equatorial plane around rotating relativistic stars such as neutron stars. First, we derive approximate analytic formulas for the angular velocity and circumferential radius at the ISCO making use of an approximate relativistic solution which is characterized by arbitrary mass, spin, mass quadrupole, current octapole and mass $2^{4}$-pole moments. Then, we show that the analytic formulas are accurate enough by comparing them with numerical results, which are obtained by analyzing the vacuum exterior around numerically computed geometries for rotating stars of polytropic equation of state. We demonstrate that contribution of mass quadrupole moment for determining the angular velocity and, in particular, the circumferential radius at the ISCO around a rapidly rotating star is as important as that of spin.

04.25.Nx, 04.40.Dg
\end{abstract}

\section{INTRODUCTION}

Observations of the low-mass X-ray binaries (LMXBs) with the Rossi X-ray Timing Explorer have revealed quasi-periodic oscillations (QPOs) around a frequency of $\sim 1 \mathrm{kHz}$ [1]. At present, more than 10 sources of $\mathrm{kHz}$ QPOs have been found [1]. One of the most impressive features of $\mathrm{kHz}$ QPOs is their very high frequency itself. The LMXBs are considered to be systems which include a neutron star of mass $M \sim 1.4 M_{\odot}$, where $M_{\odot}$ denotes the solar mass, and the accretion disk around the neutron star. The Kepler frequency of the accretion disk is

$$
f \simeq 1.185 \mathrm{kHz}\left(\frac{15 \mathrm{~km}}{R}\right)^{3 / 2}\left(\frac{M}{1.4 M_{\odot}}\right)^{1 / 2}
$$

where $R$ denotes the circumferential radius around the neutron star. If the origin of the $\mathrm{kHz}$ QPOs is certain oscillation frequencies of the accretion disk surrounding a neutron star of low magnetic field, they must be generated at less than ten Schwarzschild radii of the neutron star. This means that the $\mathrm{kHz}$ QPOs may bring us a chance to explore general relativistic effects [2].

Several authors have recently suggested that at least some of the $\mathrm{kHz}$ QPOs may be related to the Kepler frequency at the innermost stable circular orbit (ISCO) of the accretion disk around a neutron star [1]. One of the most strong reasons is that in many sources, the maximum frequency of the $\mathrm{kHz}$ QPOs is in narrow range between 1.1 and $1.2 \mathrm{kHz}$, although they are thought to have very different mass accretion rates and magnetic fields.
Due to the fact that the ISCO is determined by the property of the central neutron star, but not by the properties of the accretion disk, such as the mass accretion rate, it has been suggested that the origin of the $\mathrm{kHz}$ QPOs of the highest frequencies may be the Kepler frequency at the ISCO. If this is true, it means that we have a great opportunity to investigate general relativistic effects [3] [2].

Another remarkable feature of $\mathrm{kHz}$ QPOs is that they exhibit some evidences that in the center of their sources, rapidly rotating neutron stars are involved as follows: (1) many sources display two peaks of the $\mathrm{kHz} \mathrm{QPO}$, and the frequency difference between the twin peaks $(\Delta f)$ changes only slightly with time although the frequency of each peak changes [1]; (2) some sources which possess twin peaks also exhibit very coherent oscillations of several hundred $\mathrm{Hz}$ in X-ray bursts [1], and the frequencies change little with time during the bursts. Furthermore, they are approximately equal to or twice $\Delta f$. Since the spin frequency of a neutron star is the only candidate which changes only slightly on short time scales, the origin of the frequency difference between the twin peaks of QPOs and the oscillation frequencies in the Xray bursts seem to correspond to the spin frequencies (or twice them) of neutron stars [1]. This means that QPO sources include rapidly rotating neutron stars.

Since the ISCO is determined by the geometry around the star, it is important to ask if the geometry around a neutron star can be approximated by that around a black 
hole. If the electric charge is neutral, stationary black holes must be of the Kerr type due to the uniqueness theorem 顿. Kerr black holes have mass, spin, quadrupole moment and so on, but multipole moments higher than the quadrupole are expressed in terms of the mass $M$ and the spin angular momentum $J=S_{1}=q M^{2}(|q|<1)$ as $M_{2 l}=M(i q M)^{2 l}$ and $S_{2 l-1}=-i M(i q M)^{2 l-1}(l=$ $1,2,3, \cdots)$ 周, where $M_{l}$ and $S_{l}$ denote the mass and current moments, respectively. This means that the geometry outside the black hole horizon is expressed only in terms of $M$ and $q$. As a result, the ISCO is determined solely by them. However, this is not the case for neutron stars. In the neutron star case, multipole moments higher than and including the quadrupole do not depend on the mass and spin in such a simple manner, and they are determined when the equation of state of the neutron star is given.

The purpose of this paper is to point out the significance of the multipole moments (in particular, the mass quadrupole moment) of rapidly rotating neutron stars in determining the ISCO around them. This is due to the following fact: In the case of a Kerr black hole, the magnitude of the quadrupole moment is denoted by $M_{2}=-q^{2} M^{3}$ [5], and $\left|M_{2}\right|$ is always smaller than $M^{3}$. In this case, the effect of the quadrupole moment is not important except in the case $q \sim 1$. However, in the case of a rotating neutron star, $\left|M_{2} / q^{2} M^{3}\right|$ may be much larger than unity $\sim 10$, and hence for rapidly rotating neutron stars which seem to be located at the center of QPO sources, $\left|M_{2}\right|$ may be larger than $q M^{3}$ for the case $q \gtrsim 0.1$ [6]. In such a case, the effect due to the quadrupole moment is as important as that due to the spin.

The paper is organized as follows. In Sec. II, we derive approximate analytic formulas for the angular velocity and circumferential radius of the ISCO around a rotating object characterized by its mass, spin angular momentum, mass quadrupole, current octapole, and mass $2^{4}$-pole moments. In Sec. III, we perform numerical computations for obtaining stationary, axisymmetric spacetimes of rotating stars, and making use of the numerical results, we demonstrate that the accuracy of our formu-

\footnotetext{
* In this paper, we use units of $G=1=c$, where $G$ and $c$ are the gravitational constant and speed of light.
}

las derived in Sec. II are accurate. Sec. IV is devoted to a summary.

\section{APPROXIMATE ANALYTIC FORMULAS FOR THE ANGULAR VELOCITY AND CIRCUMFERENTIAL RADIUS AT ISCO}

\section{A. Basic equations}

The line element of the vacuum exterior outside a stationary, axisymmetric rotating object is generally written as [7]

$$
d s^{2}=-F(d t-\omega d \varphi)^{2}+\frac{1}{F}\left[e^{2 \gamma}\left(d \rho^{2}+d z^{2}\right)+\rho^{2} d \varphi^{2}\right] .
$$

Throughout this paper, we assume that the spacetime has reflection symmetry with respect to the equatorial plane $z=0$, so that $F, \omega$, and $\gamma$ are functions of $\rho$ and $|z|$.

Our purpose is to derive approximate formulas for the angular velocity and circumferential radius at the ISCO of a test particle on the equatorial plane around a rotating object. In the case when the test particle stays on the equatorial plane $(z=0)$, geodesic equations can be integrated to give

$$
\begin{aligned}
\frac{d t}{d \tau} & =\frac{E g_{\varphi \varphi}+\ell g_{t \varphi}}{g_{2}}, \\
\frac{d \varphi}{d \tau} & =\frac{E g_{t \varphi}+\ell g_{t t}}{g_{2}}, \\
g_{\rho \rho}\left(\frac{d \rho}{d \tau}\right)^{2} & =-1+E^{2} \frac{g_{\varphi \varphi}}{g_{2}}+2 E \ell \frac{g_{t \varphi}}{g_{2}}+\ell^{2} \frac{g_{t t}}{g_{2}} \\
& \equiv-V(\rho),
\end{aligned}
$$

where $E$ and $\ell$ denote the specific energy and specific angular momentum of the test particle. $g_{t t}=-F, g_{t \varphi}=$ $F \omega, g_{\varphi \varphi}=-F \omega^{2}+\rho^{2} / F$, and $g_{2} \equiv g_{t \varphi}^{2}-g_{t t} g_{\varphi \varphi}\left(g_{2}=\rho^{2}\right.$ in the present line element). For circular orbits, relations of the angular velocity $\Omega, E$, and $\ell$ are derived from the conditions $V=0=d V / d \rho$ as

$$
\begin{aligned}
& \Omega \equiv \frac{d \varphi}{d t}=\frac{-g_{t \varphi, \rho}+\sqrt{\left(g_{t \varphi, \rho}\right)^{2}-g_{t t, \rho} g_{\varphi \varphi, \rho}}}{g_{\varphi \varphi, \rho}}, \\
& E=-\frac{g_{t t}+g_{t \varphi} \Omega}{\sqrt{-g_{t t}-2 g_{t \varphi} \Omega-g_{\varphi \varphi} \Omega^{2}}}, \\
& \ell=\frac{g_{t \varphi}+g_{\varphi \varphi} \Omega}{\sqrt{-g_{t t}-2 g_{t \varphi} \Omega-g_{\varphi \varphi} \Omega^{2}}} .
\end{aligned}
$$


For simplicity, we only consider prograde orbits in this paper. A circular orbit is stable (unstable) if

$$
\frac{d^{2} V}{d \rho^{2}}=\frac{1}{g_{2}}\left(\frac{d^{2} g_{2}}{d \rho^{2}}-E^{2} \frac{d^{2} g_{\varphi \varphi}}{d \rho^{2}}-2 E \ell \frac{d^{2} g_{t \varphi}}{d \rho^{2}}-\ell^{2} \frac{d^{2} g_{t t}}{d \rho^{2}}\right)
$$

is positive (negative). Hence, the coordinate radius $\rho$ and $\Omega$ at the ISCO are determined from the condition where $d^{2} V / d \rho^{2}$ is vanishing.

Note that Eq. (2.8) is independent of metric function $\gamma$. Also, the angular velocity $\Omega$ and the circumferential radius $\left(\sqrt{g_{\varphi \varphi}} \equiv R\right)$ are independent of $\gamma$. Thus, we only need $F$ and $\omega$ in the following.

\section{B. Metric from the Ernst potential}

A stationary axisymmetric vacuum geometry is completely determined by the Ernst potential [8], which is defined as

$$
\mathcal{E}=F+i \psi=\frac{\sqrt{\rho^{2}+z^{2}}-\xi}{\sqrt{\rho^{2}+z^{2}}+\xi},
$$

where $F=-g_{t t}$ and $\xi$ is a complex potential. If we know $\psi, \omega$ is calculated as

$$
\omega=-\left.\int_{\infty}^{\rho} \frac{\rho^{\prime}}{F^{2}} \frac{\partial \psi}{\partial z} d \rho^{\prime}\right|_{\text {constant } z} .
$$

Thus, once we know $\xi$, we have all the necessary information.

$\xi$ has the property that it can be expanded as [9] [10]

$$
\xi=\sum_{j=0}^{\infty} \sum_{k=0}^{\infty} a_{2 j, k} \frac{\rho^{2 j} z^{k}}{\left(\rho^{2}+z^{2}\right)^{2 j+k}},
$$

where $a_{j, k}$ are complex constants in which information of the multipole moments of spacetime is completely contained. Note that $a_{j, k}$ is non-zero only for non-negative, even $j$ and non-negative $k$. Also, because of reflection symmetry with respect to the equatorial plane, $a_{j, k}$ is real for even $k$, and pure imaginary for odd $k$. Note that for investigation of the ISCO on the equatorial plane, we only need $a_{j, 0}$ and $a_{j, 1}$.

Fodor, Hoenselaers, and Perjes (FHP) [9] show that all the components of $a_{j, k}$ are derived by the following recursive relation

$$
\begin{aligned}
a_{r, s+2}= & \frac{1}{(s+1)(s+2)}\left[-(r+2)^{2} a_{r+2, s}\right. \\
& +\sum_{k, l, p, j} a_{k, l} a_{r-k-p, s-l-j}^{*} \times \\
& \left\{a_{p, j}\left(p^{2}+j^{2}-4 p-5 j-2 p k-2 j l-2\right)\right. \\
& +a_{p+2, j-2}(p+2)(p+2-2 k) \\
& \left.\left.+a_{p-2, j+2}(j+2)(j+1-2 l)\right\}\right],
\end{aligned}
$$

where the sum is taken for $0 \leq k \leq r, 0 \leq l \leq s+1$, $0 \leq p \leq r-k$, and $-1 \leq j \leq s-l$ [10], and $a_{j, k}^{*}$ denotes the complex conjugate of $a_{j, k}$. As pointed out in ref. [10], it can be shown that $a_{r, s+2}(s \geq 0)$ is a function of $a_{j, 0}$ and $a_{j-1,1}$ with $j \leq r+s+2$. This means that if we know $a_{j, 0}$ and $a_{j, 1}$ for $j \geq 0$, the entire spacetime metric (and of course, the metric on the equatorial plane) are completely determined. Note that if we know $a_{j, 0}$ up to $j=2 n$ and $a_{j, 1}$ up to $j=2 n-2$, we can calculate $a_{0, k}$ up to $k=2 n$. Conversely, if we know $a_{0, k}$ up to $k=2 n$, we can calculate $a_{j, 0}$ up to $j=2 n$ and $a_{j, 1}$ up to $j=2 n-2$.

In principle, we can calculate terms of $a_{j, k}$ up to arbitrary large $j$ and $k$ using Eq. (2.12). In practice, however, we have to truncate higher terms. To access an appropriate truncation point, we can make use of the solution for slowly rotating black holes of the mass $M$ and the angular momentum $J\left(=S_{1}\right) \ll M^{2}$. In this case,

$$
\xi=\frac{M \rho}{\sqrt{\rho^{2}+M^{2}}}+\frac{i z \rho J}{\left(\rho^{2}+M^{2}\right)^{3 / 2}}+O\left(z^{2}\right),
$$

where we have neglected terms of $O\left(J^{2} / M^{4}\right)$. Note that $\rho$ is related to the Schwarzschild radial coordinate $r_{s}$ as $\rho=\sqrt{r_{s}\left(r_{s}-2 M\right)}$. The Schwarzschild coordinate radius of the ISCO is $r_{s}=6 M\left(1-\sqrt{8 / 27} J / M^{2}\right)$, so that the radius of the ISCO in $\rho$ is given by

$$
\rho_{\mathrm{ISCO}}=\sqrt{24} M-\frac{10 J}{3 M} .
$$

We have investigated how the estimated values of $\rho_{\text {ISCO }}$ differ from the above one by expanding $\xi$ in terms of $O(M / \rho)$ as

$$
\begin{aligned}
\xi= & 1-\frac{M^{2}}{2 \rho^{2}}+\frac{3 M^{4}}{8 \rho^{4}}-\frac{5 M^{6}}{16 \rho^{6}}+\frac{35 M^{8}}{128 \rho^{8}}-\frac{63 M^{10}}{256 \rho^{10}} \\
& +i \frac{z J}{\rho^{2}}\left(1-\frac{3 M^{2}}{2 \rho^{2}}+\frac{15 M^{4}}{8 \rho^{4}}-\frac{35 M^{6}}{16 \rho^{6}}+\frac{315 M^{8}}{128 \rho^{8}}\right) \\
& +O\left(\rho^{-12}\right) .
\end{aligned}
$$

We have found that in the case when we take up to $O\left(\rho^{-6}\right), O\left(\rho^{-8}\right)$, and $O\left(\rho^{-10}\right)$ terms, the errors in $\rho_{\mathrm{ISCO}}$ 
are about $4 \times 10^{-4}, 10^{-5}$ and $4 \times 10^{-7}$, respectively, for the coefficient of $M$, and about $2 \times 10^{-3}, 10^{-4}$ and $4 \times 10^{-6}$, respectively, for the coefficient of $J / M$. The order of magnitude of the errors in $\Omega_{\mathrm{ISCO}}$ have turned out to be almost the same as those in $\rho_{\text {ISCO }}$. Thus, we expect that this method will generate a fairly accurate approximate formula even if we include the higher multipole moments. In the following, we take $a_{j, 0}$ up to $j=10$ and $a_{j, 1}$ up to $j=8$, i.e., we take into account all the terms up to $O\left(\rho^{-10}\right)$ consistently. In other word, we calculate $a_{0, k}$ up to $k=10$, and neglect $a_{0, k}$ of $k \geq 11$.

\section{Results}

Our strategy for determining the ISCO is as follows. First, we assume that the spacetime is characterized by mass $M$, spin angular momentum $J\left(=S_{1}\right)=q M^{2}$, mass quadrupole $M_{2}=-Q_{2} M^{3}$, current octapole moments $S_{3}=-q_{3} M^{4}$, and mass $2^{4}$-pole $M_{4}=Q_{4} M^{5}$, and neglect the higher multipole moments. Note that $q, Q_{2}, q_{3}$, and $Q_{4}$ are positive for a rotating star in a prograde spin.

In the case of a rotating neutron star, we may assume $q \sim O\left(\epsilon_{1}\right), Q_{2} \sim O\left(\epsilon_{2}\right), q_{3} \sim O\left(\epsilon_{1} \epsilon_{2}\right), Q_{4} \sim O\left(\epsilon_{2}^{2}\right)$, where $\epsilon_{1}, \epsilon_{2} \ll 1$, because $q$ and $Q_{2}$ are expected to be less than unity. For a slowly rotating neutron star, $Q_{2} \sim O\left(q^{2}\right)$, so that $\epsilon_{2} \sim O\left(\epsilon_{1}^{2}\right)$. In this case, $Q_{2} \sim O\left(\epsilon_{1}^{2}\right), q_{3} \sim O\left(\epsilon_{1}^{3}\right)$, $Q_{4} \sim O\left(\epsilon_{1}^{4}\right)$. However, for a rapidly rotating neutron star, $Q_{2}$ can be as large as $q$, so that whenever the terms proportional to $q^{2}$ make a significant contribution, we should also take into account the terms proportional to $Q_{2}^{2}, Q_{4}, q q_{3}$, and so on. This is the reason why we take into account $S_{3}$ and $M_{4}$.

Hereafter, we expand all the quantities by means of $\epsilon \equiv \epsilon_{1}$ by formally setting $q \rightarrow \epsilon q, Q_{2} \rightarrow \epsilon^{2} Q_{2}, q_{3} \rightarrow$ $\epsilon^{3} q_{3}$, and $Q_{4} \rightarrow \epsilon^{4} Q_{4}$, and retain all the terms up to $O\left(\epsilon^{4}\right)$ consistently. Thus, the formulas derived below are accurate up to $O\left(\epsilon_{1}^{4}\right)$ for a slowly rotating neutron star. Even for a rapidly rotating neutron star, the formulas include all the terms of $O\left(\epsilon_{1}\right), O\left(\epsilon_{2}\right), O\left(\epsilon_{1}^{2}\right), O\left(\epsilon_{2}^{2}\right)$, and $O\left(\epsilon_{1} \epsilon_{2}\right)$. Hence, they are still accurate to $O\left(Q_{2}^{2}\right)$.

As mentioned in the previous subsection, we need $a_{0, k}$ up to $k=10$. Relation between multipole moments and $a_{0, k}$ for $0 \leq k \leq 10$ have been already given by FHP [9]. Hence, by using them, we can calculate $a_{2 j, 0}(1 \leq j \leq 5)$ and $a_{2 j, 1}(1 \leq j \leq 4)$ up to $O\left(\epsilon^{4}\right)$ using Eq. (2.12) [11. (The explicit forms of $a_{0, k}, a_{2 j, 0}$ and $a_{2 j, 1}$ are shown in
Appendix A). Once $\xi$ is determined up to $O\left(\rho^{-10}\right), F$ and $\omega$ are straightforwardly obtained from Eqs. 2.9) and (2.10) in the following form;

$$
\begin{aligned}
& F=1+\sum_{j=1}^{11} C_{F, j}\left(\frac{M}{\rho}\right)^{j}+O\left(\rho^{-12}\right), \\
& \omega=\sum_{j=3}^{11} C_{\omega, j} \frac{M^{j-1}}{\rho^{j}}+O\left(\rho^{-12}\right),
\end{aligned}
$$

where $C_{F, j}$ and $C_{\omega, j}$ are functions of $q, Q_{2}, q_{3}$, and $Q_{4}$. Substituting these equations into $d^{2} V / d \rho^{2}=0$, we rewrite Eq. (2.8) in the form

$$
\sum_{i=0}^{4} \epsilon^{i} A_{i}(\rho)=0,
$$

where the coefficients $A_{i}$ are independent of $\epsilon$ but depend on $q, Q_{2}, q_{3}, Q_{4}$, and $\rho$. These coefficients can be explicitly written out by substituting Eqs. (2.16) and (2.17) into Eq. 2.8) (making use of Eqs. (2.6) and (2.7)) and gathering in powers of $\epsilon$. We then look for a solution in the form

$$
\rho=\sum_{i=0}^{4} \epsilon^{i} c_{i},
$$

where we know that $c_{0}=\sqrt{24} M$ and $c_{1}=-10 q M / 3$. Imposing that Eq. (2.18) holds in each order of $\epsilon$, it is rewritten into three algebraic equations for $c_{2}, c_{3}$, and $c_{4}$ as

$$
\begin{gathered}
\frac{1}{2} \frac{d^{2} A_{0}}{d \rho^{2}} c_{1}^{2}+\frac{d A_{0}}{d \rho} c_{2}+\frac{d A_{1}}{d \rho} c_{1}+A_{2}=0, \\
\frac{1}{6} \frac{d^{3} A_{0}}{d \rho^{3}} c_{1}^{3}+\frac{d^{2} A_{0}}{d \rho^{2}} c_{1} c_{2}+\frac{d A_{0}}{d \rho} c_{3} \\
+\frac{1}{2} \frac{d^{2} A_{1}}{d \rho^{2}} c_{1}^{2}+\frac{d A_{1}}{d \rho} c_{2}+\frac{d A_{2}}{d \rho} c_{1}+A_{3}=0, \\
\frac{1}{24} \frac{d^{4} A_{0}}{d \rho^{4}} c_{1}^{4}+\frac{1}{2} \frac{d^{3} A_{0}}{d \rho^{3}} c_{1}^{2} c_{2}+\frac{1}{2} \frac{d^{2} A_{0}}{d \rho^{2}}\left(c_{2}^{2}+2 c_{1} c_{3}\right) \\
+\frac{d A_{0}}{d \rho} c_{4}+\frac{1}{6} \frac{d^{3} A_{1}}{d \rho^{3}} c_{1}^{3}+\frac{d^{2} A_{1}}{d \rho^{2}} c_{1} c_{2}+\frac{d A_{1}}{d \rho} c_{3} \\
+\frac{1}{2} \frac{d^{2} A_{2}}{d \rho^{2}} c_{1}^{2}+\frac{d A_{2}}{d \rho} c_{2}+\frac{d A_{3}}{d \rho} c_{1}+A_{4}=0,
\end{gathered}
$$

where $A_{k}$ and their derivatives are evaluated at $\rho=c_{0}$. From these algebraic equations we obtain

$$
\begin{aligned}
c_{2} & \simeq\left(-1.41671 q^{2}+1.07648 Q_{2}\right) M, \\
c_{3} \simeq & \left(-1.45081 q^{3}+1.60313 q Q_{2}-0.32561 q_{3}\right) M, \\
c_{4} \simeq & \left(-1.87941 q^{4}+2.74953 q^{2} Q_{2}-0.37568 Q_{2}^{2}\right. \\
& \left.\quad+0.09377 Q_{4}-0.70086 q q_{3}\right) M .
\end{aligned}
$$


Using these approximate solutions, we reach approximate expressions for the angular velocity and circumferential radius of the ISCO, $\Omega_{\mathrm{ISCO}}$ and $R_{\mathrm{ISCO}}$, as follows.

$$
\begin{aligned}
& \Omega_{\mathrm{ISCO}}=\frac{1}{6 \sqrt{6} M}\left(1+0.74846 q+0.78059 q^{2}-0.23429 Q_{2}\right. \\
&+ 0.98094 q^{3}-0.64406 q Q_{2}+0.07432 q_{3} \\
&+1.38118 q^{4}-1.41729 q^{2} Q_{2}+0.12798 Q_{2}^{2} \\
&\left.-0.02129 Q_{4}+0.25028 q q_{3}\right), \\
& R_{\mathrm{ISCO}}=6 M\left(1-0.54433 q-0.22619 q^{2}+0.17989 Q_{2}\right. \\
&-0.23002 q^{3}+0.26296 q Q_{2}-0.05317 q_{3} \\
&-0.29693 q^{4}+0.44546 q^{2} Q_{2}-0.06249 Q_{2}^{2} \\
&+\left.0.01544 Q_{4}-0.11310 q q_{3}\right) .
\end{aligned}
$$

For the case of Kerr metric, $Q_{2}=q^{2}, q_{3}=q^{3}$, and $Q_{4}=$ $q^{4}$, we obtain

$$
\begin{gathered}
\Omega_{\mathrm{ISCO}}=\frac{1}{6 \sqrt{6} M}\left(1+0.74846 q+0.54630 q^{2}+0.41120 q^{3}\right. \\
\left.+0.32085 q^{4}\right), \\
R_{\mathrm{ISCO}}=6 M\left(1-0.54433 q-0.04630 q^{2}-0.02023 q^{3}\right. \\
\left.-0.01162 q^{4}\right) .
\end{gathered}
$$

On the other hand, the exact Kerr solution gives 12

$$
\begin{gathered}
\Omega_{\mathrm{ISCO}}^{\mathrm{Kerr}}=\frac{1}{6 \sqrt{6} M}\left(1+0.74846 q+0.54630 q^{2}+0.41108 q^{3}\right. \\
\left.+0.31991 q^{4}+O\left(q^{5}\right)\right), \\
R_{\mathrm{ISCO}}^{\mathrm{Kerr}}=6 M\left(1-0.54433 q-0.04630 q^{2}-0.02016 q^{3}\right. \\
\left.-0.01110 q^{4}+O\left(q^{5}\right)\right) .
\end{gathered}
$$

Thus, the error of the coefficients in our approximate formulas of $\Omega_{\mathrm{ISCO}}$ and $R_{\mathrm{ISCO}}$ is less than $10^{-4}$ for $O\left(\epsilon^{2}\right)$ terms, less than $10^{-3}$ for $O\left(\epsilon^{3}\right)$ terms, and $\sim 10^{-2}$ for $O\left(\epsilon^{4}\right)$ terms. Hence, for slowly rotating neutron stars $(q<0.1)$, our formulas are accurate enough. Even for very rapidly rotating neutron stars of $q, Q_{2} \sim 0.5$, we may expect that they will yield very accurate values.

We note that in Eqs. (2.24) and (2.25), the signs of coefficients of the terms including $Q_{2}$ such as $Q_{2}, q Q_{2}$ and $q^{2} Q_{2}$ are opposite to those of $q^{k}(k=1-4)$ terms. As shown in ref. [6], $Q_{2} / q^{2}$ is always larger than unity for a rotating neutron star, and hence $Q_{2}$ may be of $O(q)$ for rapidly rotating neutron stars of $q \gtrsim 0.1$. This implies that for rapidly rotating neutron stars, the effect due to the $q^{n}$ terms is significantly suppressed by that due to
$Q_{2}$. Thus, we conclude that the effect of the quadrupole is important in determining the ISCO even when $q \sim$ 0.1. On the other hand, the coefficients of $q_{3}$ and $Q_{4}$ are smaller than those of $q$ and $Q_{2}$. Thus, their contributions are small.

The formulas of $E$ and $\ell$ at the ISCO are shown in Appendix B.

\section{NUMERICAL STUDY}

To confirm the accuracy of the formulas derived in Sec. II, we compare them with numerical solutions. We numerically construct stationary, axisymmetric spacetimes of relativistic rotating stars with a polytropic equation of state using Komatsu, Eriguchi, and Hachisu method [13. Then, we estimate $\Omega_{\mathrm{ISCO}}$ and $R_{\mathrm{ISCO}}$ as well as the multipole moments of the numerically generated spacetimes. It is desirable to include all the multipole moments up to $Q_{4}$, and compare our analytic formulas with the numerical results. However, accurate numerical calculation of $Q_{4}$ is difficult. In this section, we simply set $Q_{4}=\alpha Q_{2}^{4}$. For the Newtonian incompressible case (Maclaurin spheroid case), $\alpha=15 / 7$, and for Newtonian compressible case, we find $\alpha<15 / 7$. Due to general relativistic effects, stars are more centrally condensed than those in the Newtonian case, so that we expect that $\alpha$ in general relativity is smaller than that in the Newtonian theory, i.e., $0<\alpha \lesssim 2$. Hence, we simply set $\alpha=1$. Fortunately, the coefficients of $Q_{4}$ in $\Omega_{\mathrm{ISCO}}$ and $R_{\mathrm{ISCO}}$ are small, so that our rough treatment does not affect the result much. In fact, we also set $\alpha=0$ and 2 , but our conclusion is not changed at all.

\section{A. Basic equations}

We consider the energy momentum tensor of an ideal fluid,

$$
T_{\mu \sigma}=\rho_{b}\left(1+\varepsilon+\frac{P}{\rho_{b}}\right) u_{\mu} u_{\sigma}+P g_{\mu \sigma},
$$

where $\rho_{b}, P, \varepsilon, u^{\mu}$, and $g_{\mu \sigma}$ are the baryon rest mass density, pressure, specific internal energy, four velocity, and spacetime metric. We adopt the polytropic equation of state,

$$
P=K \rho_{b}^{\Gamma}=(\Gamma-1) \rho_{b} \varepsilon ; \Gamma=1+\frac{1}{n},
$$


where $K$ and $n$ are the polytropic constant and the polytropic index.

We only consider the case when stars uniformly rotate around the $z$-axis. Hence, we set $u^{r}=u^{\theta}=0$ and $u^{\varphi}=$ $u^{0} \Omega_{s}$, where $\Omega_{s}$ is the spin angular velocity of the rotating star. In this case, the fluid equations of motion are easily integrated to give [14]

$$
\begin{aligned}
\frac{h^{2}}{\left(u^{0}\right)^{2}} & =-h^{2}\left(g_{t t}+2 g_{t \varphi} \Omega_{s}+g_{\varphi \varphi} \Omega_{s}^{2}\right) \\
& =\text { constant }
\end{aligned}
$$

where $h=1+\varepsilon+P / \rho_{b}=1+\Gamma \varepsilon$.

Following Butterworth and Ipser [15], we write the line element as

$$
\begin{aligned}
d s^{2}= & -e^{2 \nu} d t^{2}+r^{2} \sin ^{2} \theta B^{2} e^{-2 \nu}(d \varphi-\omega d t)^{2} \\
& +e^{2 \zeta-2 \nu}\left(d r^{2}+r^{2} d \theta^{2}\right),
\end{aligned}
$$

where $\nu, B, \zeta$, and $\omega$ are field functions depending on $r$ and $\theta$. We present the field equations for these variables and briefly describe our numerical method in Appendix C.

Once the field variables are computed, the ISCO on the equatorial plane is found making use of Eq. (2.8) together with Eqs. 2.5) -(2.7). To examine the accuracy of our approximate analytic formulas derived in Sec. II, we also need to estimate the multipole moments. For $M$ and $q$, we have the formulas as 15

$$
\begin{gathered}
M=\int d^{3} x\left[e^{2 \zeta} \rho_{b} B\left[2 h\left(u^{0}\right)^{2}-e^{-2 \nu}\{1+\varepsilon(2-\Gamma)\}\right]\right. \\
\left.+2 r^{2} \sin ^{2} \theta\left(\Omega_{s}-\omega\right) \omega B^{3}\left(u^{0}\right)^{2} e^{2 \zeta-4 \nu} \rho_{b} h\right] \\
q=\frac{1}{M^{2}} \int d^{3} x r^{2} \sin ^{2} \theta\left(\Omega_{s}-\omega\right) B^{3}\left(u^{0}\right)^{2} e^{2 \zeta-4 \nu} \rho_{b} h
\end{gathered}
$$

For $Q_{2}$ and $q_{3}$, we estimate them by using the asymptotic behaviors of $\nu$ and $\omega$. For $r \rightarrow \infty, \nu, \omega$ and $B$ behaves as 15]

$$
\begin{aligned}
\nu \rightarrow- & \frac{M}{r}+\frac{B_{0} M}{3 r^{3}}+\frac{Q_{2} M^{3}}{r^{3}} P_{2}(y)+O\left(r^{-4}\right), \\
\omega \rightarrow & \frac{2 q M^{2}}{r^{3}}-\frac{6 q M^{3}}{r^{4}}+\frac{6}{5}\left[8-\frac{3 B_{0}}{M^{2}}\right] \frac{q M^{4}}{r^{5}} \\
& \quad-\frac{q_{3} M^{4}}{r^{5}}\left(5 y^{2}-1\right)+O\left(r^{-6}\right) \\
B \rightarrow 1 & +\frac{B_{0}}{r^{2}}+O\left(r^{-4}\right)
\end{aligned}
$$

where $y=\cos \theta, P_{2}(y)=\left(3 y^{2}-1\right) / 2$, and $B_{0}$ is a constant which is $-M^{2} / 4$ for spherical cases. When we numerically obtain $\nu, \omega$, and $B$, we can extract information of $Q_{2}$ and $q_{3}$ at a large radius $r \gg M$ near the outer numerical boundary $\left(r=r_{\max }\right)$ as

$$
\begin{aligned}
& Q_{2}=5 \frac{r^{3}}{M^{3}} \int_{0}^{1} d y P_{2}(y) \nu \\
& q_{3}=-\frac{21 r^{5}}{16 M^{4}} \int_{0}^{1} d y\left(5 y^{2}-1\right)\left(1-y^{2}\right) \omega .
\end{aligned}
$$

We evaluate $Q_{2}$ and $q_{3}$ at various large radii, and find that $Q_{2}$ quickly converges as $r \rightarrow r_{\max }$. This suggests that the error for estimation of $Q_{2}$ is very small (we guess it is less than 1\%). This is mainly because the coefficient of $O\left(r^{-4}\right)$ part in $\nu$ is not large. On the other hand, convergence of $q_{3}$ at $r \rightarrow r_{\max }$ is slow because the coefficient of $O\left(r^{-6}\right)$ part in $\omega$ is large [15]. To obtain $q_{3}$ accurately, it is desirable to attach the outer numerical boundary as $r_{\max } \gg M$, but to do that we need to take many grid points and hence need a long computational time. To save computational time, we use here an extrapolation method to estimate $q_{3}$; i.e., we calculate $q_{3}$ at several large radii which are not near the outer boundary $\left(r \sim 3 r_{\max } / 4\right)$, and extrapolate true value of $q_{3}$ at $r \rightarrow \infty$ by assuming that $q_{3}$ behaves as $q_{3}(r)=q_{3}(\infty)+C / r$ for the large radii, where $C$ is a constant. Since this method is rough, we guess that the error of $q_{3}$ may be $\sim 10 \%$ in this method. However, the large error in $q_{3}$ does not affect the following analysis much because the coefficients of $q_{3}$ terms in $\Omega_{\mathrm{ISCO}}$ and $R_{\mathrm{ISCO}}$ are fortunately small. The important quantities in our analysis are $M, q$ and $Q_{2}$. We mention that we have also evaluated $M$ and $q$ by using

$$
\begin{aligned}
M= & -r \int_{0}^{1} d y \nu \\
q=\left[1-\frac{3 M}{r}+\right. & \left.\frac{3}{5}\left(8-\frac{3 B_{0}}{M^{2}}\right)\right]^{-1} \frac{3 r^{3}}{4 M^{2}} \\
& \times \int_{0}^{1} d y\left(1-y^{2}\right) \omega,
\end{aligned}
$$

and confirmed that $M$ calculated by Eqs. (3.5) and (3.12) and $J$ calculated by Eqs. (3.6) and (3.13), respectively, agree very well.

\section{B. Results}

As the polytropic index, we set $n=1$. In Fig. 1, we show the relation between $\rho_{b, 0}\left(\rho_{b}\right.$ at $\left.r=0\right)$ and $M$ for the case $n=1$. Note that in the figure, we plot non-dimensional quantities $\rho_{b, 0} K^{n}$ and $M K^{-n / 2}$. The 
lower and upper solid lines denote the relations for the spherical star and for the rotating star at the mass shed limit, respectively. The filled squares denote data sets that are used for comparison with our analytic formulas. The dotted line is the critical line above which the ISCO ceases to exist. For sufficiently large $\rho_{b, 0}$, stars are unstable against radial gravitational collapse [16]. The dashed line divides the stable and unstable branches: The lefthand side of it is the stable region. (Here, for judging the stability against the gravitational collapse, we have applied the turning point method shown in ref. [17].) Since unstable stars are not realized in nature, we exclude numerical data in the unstable region.

In Table I, we show all the data we use in our analysis. As pointed out in ref. [6], $Q_{2} / q^{2}$ is always greater than unity. (For the data in Table I, $2 \lesssim Q_{2} / q^{2} \lesssim 4$. )

In Fig. 2, we show

$$
\begin{aligned}
& \Delta_{\Omega}(l)=6 \sqrt{6} M\left(\Omega_{\mathrm{ISCO}}^{\text {numerical }}-\Omega_{\mathrm{ISCO}}(l)\right), \\
& \Delta_{r}(l)=\frac{1}{6 M}\left(R_{\mathrm{ISCO}}^{\text {numerical }}-R_{\mathrm{ISCO}}(l)\right),
\end{aligned}
$$

as a function of $Q_{2}$. Here, $\Omega_{\mathrm{ISCO}}(l)$ and $R_{\mathrm{ISCO}}(l)(1 \leq$ $l \leq 4)$ denote the analytic formulas in which we include terms up to $O\left(\epsilon^{l}\right)$. We also define $\Delta_{\Omega}^{\text {Kerr }}$ and $\Delta_{r}^{\text {Kerr }}$ in which we use the analytic relations for the Kerr metric. The open circles, crosses, filled circles, open triangles and open squares denote $\Delta_{a}^{\text {Kerr }}, \Delta_{a}(1), \Delta_{a}(2), \Delta_{a}(3)$ and $\Delta_{a}(4)(a=\Omega$ or $r)$, respectively.

From Fig.2, we find apparently that the Kerr formulas for the ISCO are not appropriate at all. The formula $R_{\mathrm{ISCO}}(1)$ is as bad as the Kerr formula, but $\Omega_{\mathrm{ISCO}}(1)$ is fairly good. The latter feature seems accidental: Since the relation $Q_{2} \sim \alpha q^{2}$ with $\alpha \sim 2-4$ holds, cancellations between $q^{2}$ and $Q_{2}$ terms, between $q^{3}$ and $q Q_{2}$ terms, and among $q^{4}, q^{2} Q_{2}$ and $Q_{4}$ terms occur. (In contrast, if $Q_{2} \sim q^{2}, R_{\mathrm{ISCO}}(1)$ will be bad, while $\Omega_{\mathrm{ISCO}}(1)$ will be good. If $Q_{2} \sim 10 q^{2}$, both formulas will not be good at all).

It should be stressed that for small $Q_{2}, \Delta_{\Omega}^{\text {Kerr }}$ and $\Delta_{r}^{\text {Kerr }}$ (also $\Delta_{r}(1)$ ) are roughly proportional to $Q_{2}$, while $\Delta_{\Omega}(2)$ and $\Delta_{r}(2)$ remain very small. Recalling that in the Kerr formulas $\Omega_{\mathrm{ISCO}}^{\mathrm{Kerr}}$ and $R_{\mathrm{ISCO}}^{\mathrm{Kerr}}$, the effect of $Q_{2}$ as an independent variable is absent, this feature implies a substantial effect of $Q_{2}$ on the determination of the ISCO around neutron stars even for $q \sim 0.1$.

We find the errors of the formulas $\Omega_{\mathrm{ISCO}}(2)$ and
$R_{\mathrm{ISCO}}(2)$ are always very small for small $Q_{2}<0.2$ ( $q \lesssim 0.2$ in this case). For large $Q_{2}>0.2$, however, the errors gradually increase. This indicates that the effect of the terms of $O\left(Q_{2}^{2}\right)$ (such as $Q_{4}$ ) is not negligible. Therefore, it should be necessary to correctly take into account the terms of $O\left(Q_{2}^{2}\right)$, in order to give an appropriate formula for very rapidly rotating neutron stars. Note, however, that Fig. 2 also indicates that $\Delta_{a}(a=\Omega$ or $r)$ adequately converges to zero by adding higher order terms in $\epsilon$. Thus, unless $q, Q_{2} \gtrsim 1$, an appropriate formula for very rapidly rotating neutron stars of large $q$ and $Q_{2}$ can be derived along the line presented in this paper.

\section{SUMMARY}

In this paper, we have investigated the ISCO of a test particle moving on the equatorial plane around a rotating object. We have derived fairly accurate approximate formulas for the angular velocity and circumferential radius of the ISCO including mass, spin, quadrupole, current octapole, mass $2^{4}$-pole moments of a rotating object. Our formulas show that the effect of quadrupole moment is important for determining the angular velocity and, in particular, the circumferential radius of the ISCO for rapidly rotating neutron stars of $Q_{2} \gtrsim q[6]$.

In a recent paper, Miller, Lamb, and Cook 2] performed a numerical computation for analyzing the ISCO around rapidly rotating neutron stars, and pointed out that $\Omega_{\mathrm{ISCO}}$ and in particular $R_{\mathrm{ISCO}}$ are not correctly determined by the first order analytic treatment in $q$. Our present study clarifies the reason for inaccuracy of the first order formula which is mainly due to the neglection of the quadrupole moment.

\section{ACKNOWLEDGMENTS}

Numerical computation was in part performed using FACOM VX/4R in the data processing center of National Astronomical Observatory of Japan (NAOJ). This work was in part supported by a Japanese Grant-in-Aid of Ministry of Education, Culture, Science and Sports (Nos. 08NP0801 and 09740336). 


\section{APPENDIX A: COEFFICIENTS FOR THE ERNST POTENTIAL}

FHP [9] give

$$
\begin{aligned}
a_{0,0}= & M, \quad a_{0,1}=i J, \quad a_{0,2}=M_{2}, \\
a_{0,3}= & i S_{3}, \quad a_{0,4}=M_{4}+\frac{M_{20} M}{7}, \\
a_{0,5}= & -i \frac{J M_{20}}{21}+\frac{M_{30} M}{3}, \\
a_{0,6}= & -\frac{M_{20} M^{3}}{33}+\frac{5 M_{20} M_{2}}{231}-\frac{4 i M_{30} J}{33}+\frac{8 M_{31} M}{33} \\
& \quad+\frac{6 M_{40} M}{11}, \\
a_{0,7}= & \frac{19 i M_{20} M^{2} J}{429}-\frac{15 M_{30} M^{3}}{143}+M^{8} O\left(\epsilon^{5}\right), \\
a_{0,8}= & \frac{M_{20} M^{5}}{143}+\frac{53 M_{20} M^{2}}{3003}-\frac{311 M_{20} M_{2} M^{2}}{3003} \\
+ & \frac{36 i M_{30} M^{2} J}{143}-\frac{3 M^{3}}{13}\left(M_{31}+M_{40}\right)+M^{9} O\left(\epsilon^{5}\right), \\
a_{0,9}= & -\frac{43 i M_{20} M^{4} J}{2431}+\frac{7 M_{30} M^{5}}{221}+M^{10} O\left(\epsilon^{5}\right), \\
a_{0,10}= & -\frac{7 M_{20} M^{7}}{4199}+\frac{4423 M_{20} M^{4} M_{2}}{138567}-\frac{202 M_{20} M^{3} J^{2}}{12597} \\
- & \frac{462 i M_{30} M^{4} J}{4199}+\frac{28 M^{5}}{323}\left(M_{31}+M_{40}\right) \\
+ & M^{11} O\left(\epsilon^{5}\right),
\end{aligned}
$$

where $M_{20}=M M_{2}+J^{2}, M_{30}=i\left(S_{3} M-M_{2} J\right), M_{31}=$ $-S_{3} J-M_{2}^{2}$, and $M_{40}=M_{4} M+S_{3} J$ [9].

Using Eq. 2.12), $a_{2 j, 0}(1 \leq j \leq 5)$ and $a_{2 j, 1}(1 \leq j \leq$ 4) up to $O\left(\epsilon^{4}\right)$ are calculated as

$$
\begin{aligned}
a_{2,0}= & -\frac{M^{3}}{2}\left(1-Q_{2}\right), \\
a_{4,0}= & \frac{M^{5}}{56}\left(21+10 q^{2}-52 Q_{2}+21 Q_{4}\right), \\
a_{6,0}= & M^{7}\left(-\frac{5}{16}-\frac{313 q^{2}}{924}+\frac{4717 Q_{2}}{3696}\right. \\
+ & \left.\frac{289 q^{2} Q_{2}}{1848}-\frac{38 Q_{2}^{2}}{77}-\frac{151 Q_{4}}{176}+\frac{17 q_{3} q}{66}\right), \\
a_{8,0}= & M^{9}\left(\frac{35}{128}+\frac{31303 q^{2}}{64064}-\frac{101373 Q_{2}}{64064}\right. \\
& +\frac{32171 q^{4}}{384384}-\frac{22513 q^{2} Q_{2}}{27456}+\frac{57535 Q_{2}^{2}}{34944} \\
& \left.+\frac{5571 Q_{4}}{4576}-\frac{3343 q_{3} q}{6864}\right), \\
a_{10,0}= & M^{11}\left(-\frac{63}{256}-\frac{12974415 q^{2}}{20692672}+\frac{153744405 Q_{2}}{82770688}\right. \\
- & \frac{6074105 q^{4}}{22284416}+\frac{1686890165 q^{2} Q_{2}}{869092224}-\frac{2883979925 Q_{2}^{2}}{869092224}
\end{aligned}
$$

$$
\left.-\frac{1154583 Q_{4}}{739024}+\frac{2221047 q_{3} q}{2956096}\right)
$$

and

$$
\begin{aligned}
a_{2,1} & =\frac{3 i M^{4}}{2}\left(-q+q_{3}\right), \\
a_{4,1} & =i M^{6}\left(\frac{15 q}{8}+\frac{15 q^{3}}{28}-\frac{29 q Q_{2}}{28}-\frac{13 q_{3}}{4}\right), \\
a_{6,1} & =i M^{8}\left(-\frac{35 q}{16}-\frac{3763 q^{3}}{2002}+\frac{95213 q Q_{2}}{24024}+\frac{30743 q_{3}}{6864}\right), \\
a_{8,1} & =i M^{10}\left(\frac{315 q}{128}+\frac{579957 q^{3}}{155584}\right. \\
& \left.\quad-\frac{154403 q Q_{2}}{19448}-\frac{876263 q_{3}}{155584}\right) .
\end{aligned}
$$

\section{APPENDIX B: FORMULAS OF $E$ AND $\ell$ AT THE ISCO}

From Eqs. (2.6) and (2.7) with an approximate formula of $\rho_{\mathrm{ISCO}}$ shown in Sec. II, $E$ and $\ell$ at the ISCO are derived as

$$
\begin{aligned}
E_{\mathrm{ISCO}}= & 0.94281-0.03208 q-0.02975 q^{2}+0.00794 Q_{2} \\
& -0.0341 q^{3}+0.0198 q Q_{2}-0.0019 q_{3} \\
& -0.0440 q^{4}+0.0404 q^{2} Q_{2}-0.0033 Q_{2}^{2} \\
& +0.0005 Q_{4}-0.0062 q q_{3}, \\
\frac{\ell_{\mathrm{ISCO}}}{M}= & 3.4641-0.9428 q-0.4444 q^{2}+0.1879 Q_{2} \\
& -0.3953 q^{3}+0.2996 q Q_{2}-0.0392 q_{3} \\
& -0.4470 q^{4}+0.4944 q^{2} Q_{2}-0.0505 Q_{2}^{2} \\
& +0.0093 Q_{4}-0.0926 q q_{3} .
\end{aligned}
$$

On the other hand, exact solutions for the Kerr case 12] are expanded as

$$
\begin{aligned}
E_{\mathrm{ISCO}}^{\mathrm{Kerr}}= & 0.94281-0.03208 q-0.02182 q^{2} \\
& -0.01633 q^{3}-0.01294 q^{4} \\
\frac{\ell_{\mathrm{ISCO}}^{\mathrm{Kerr}}}{M}= & 3.46410-0.94281 q-0.25660 q^{2} \\
& -0.13531 q^{3}-0.08791 q^{4} .
\end{aligned}
$$

Hence, as in the case of $\Omega_{\mathrm{ISCO}}$ and $R_{\mathrm{ISCO}}$, the error of coefficients is less than $10^{-4}$ for $O\left(\epsilon^{2}\right)$ terms, $\sim 10^{-3}$ for $O\left(\epsilon^{3}\right)$ terms, and $\sim 10^{-2}$ for $O\left(\epsilon^{4}\right)$ terms.

If accretion of matter occurs from the ISCO to the central rotating body, the value of $q$ of the central star increases as 


$$
\delta q=\frac{\mu}{M}\left(\frac{\ell_{\mathrm{ISCO}}}{M}-2 q E_{\mathrm{ISCO}}\right),
$$

where $\mu$ is the rest mass of the accreting matter. Using Eqs. (B1) and (B2), an approximate formula for $\delta q$ is given as

$$
\begin{aligned}
\delta q=\frac{\mu}{M} & \left(3.4641-2.8284 q-0.3802 q^{2}+0.1879 Q_{2}\right. \\
& -0.3358 q^{3}+0.2837 q Q_{2}-0.0392 q_{3} \\
& -0.3788 q^{4}+0.4548 q^{2} Q_{2}-0.0505 Q_{2}^{2} \\
& \left.+0.0093 Q_{4}-0.0887 q q_{3}\right) .
\end{aligned}
$$

Thus we obtain the following conclusions from Eqs. (B1), (B2), and (B6): If accretion of matter occurs from the ISCO, the effect of $Q_{2}$ are that (1) the maximum energy release efficiency of accreting matter slightly decreases, and that (2) the rate of increase in the angular momentum and $\delta q$ of central body is increased.

\section{APPENDIX C: FIELD EQUATIONS FOR COMPUTING ROTATING STARS AND THE NUMERICAL METHOD}

In this appendix, we show field equation for $\nu, \omega, B$, and $\zeta$ for obtaining axially symmetric rotating stars, and briefly mentioned our numerical method to solve them. The field equations are as follows 15 .

$$
\begin{aligned}
\nu_{, r r}+ & \frac{2}{r} \nu_{, r}+\frac{1}{r^{2}}\left\{\nu_{, y y}\left(1-y^{2}\right)-2 y \nu_{, y}\right\} \\
= & 4 \pi e^{2 \zeta} \rho_{b}\left[2 h\left(u^{0}\right)^{2}-\{1+(2-\Gamma) \varepsilon\} e^{-2 \nu}\right] \\
& +\frac{1}{2} e^{-4 \nu} B^{2}\left(1-y^{2}\right)\left\{r^{2} \omega_{, r}^{2}+\omega_{, y}^{2}\left(1-y^{2}\right)\right\} \\
& -\frac{B, r}{B} \nu_{, r}-\frac{1}{B r^{2}} B_{, y} \nu_{, y}\left(1-y^{2}\right), \\
\hat{\omega}_{, r r}+ & \frac{4}{r} \hat{\omega}_{, r}+\frac{1}{r^{2}}\left\{\hat{\omega}_{, y y}\left(1-y^{2}\right)-4 y \hat{\omega}_{, y}\right\} \\
= & -16 \pi e^{2 \zeta} \rho_{b} h\left(u^{0}\right)^{2}(1-\hat{\omega})+\left(4 \nu_{, r}-\frac{3 B, r}{B}\right) \hat{\omega}_{, r} \\
& +\frac{1-y^{2}}{r^{2}}\left(4 \nu_{, y}-\frac{3 B, y}{B}\right) \hat{\omega}_{, y}, \\
B_{, r r}+ & \frac{3}{r} B_{, r}+\frac{1}{r^{2}}\left\{B, y y\left(1-y^{2}\right)-3 y B_{, y}\right\} \\
= & 16 \pi B e^{2 \zeta-2 \nu} P, \\
\xi_{, y}= & {\left[\left(B+B B_{, r} r\right)^{2}\left(1-y^{2}\right)+\left\{B y-B, y\left(1-y^{2}\right)\right\}^{2}\right]^{-1} } \\
{[(} & +B \\
& \left.+r B_{, r} r\right)\left\{-e^{-4 \nu}\left(1-\omega_{, y} \omega_{, r} r^{3} B^{3}\left(1-y^{2}\right)^{2} / 2\right.\right. \\
& -\frac{1}{2}\left\{B y-B y+2 \nu_{, y} \nu_{, r} r B\left(1-y^{2}\right)\right\}
\end{aligned}
$$

$$
\begin{aligned}
& \left.-\omega_{, y}^{2}\left(1-y^{2}\right)\right) / 2-B_{, r r} r^{2}-B_{, r} r-2 B \nu_{, r}^{2} r^{2} \\
& \left.\left.+B_{, y y}\left(1-y^{2}\right)-3 y B_{, y}+2 \nu_{, y}^{2}\left(1-y^{2}\right) B\right\}\right]
\end{aligned}
$$

where instead of $\omega$, we introduce $\hat{\omega} \equiv \omega \Omega_{s}^{-1}$ because $\omega \propto$ $\Omega_{s}$ for slowly rotating cases.

We solve Poisson type equations for $\nu, \omega(\hat{\omega})$, and $B$ as the boundary value problem. That is, we solve these equations imposing boundary conditions (3.7) - 3.9) at $r=r_{\max }>10 M$, regularity condition at $r=0$ and $\theta=0$, and reflection symmetry conditions at $\theta=\pi / 2$. On the other hand, the equation for $\zeta$ is solved using second order Runge-Kutta method imposing the boundary condition at $\theta=0$ as $\zeta=\ln B$ [15.

Using field variables, Eq. (3.3) is rewritten as

$$
\begin{aligned}
& \left(1+n K \rho_{b}^{1 / n}\right)^{2}\left[-e^{2 \nu}+\Omega_{s}^{2}(1-\hat{\omega})^{2} B^{2} e^{-2 \nu} r^{2} \sin ^{2} \theta\right] \\
& =C(=\text { constant }) .
\end{aligned}
$$

Here, $C, K$, and $\Omega_{s}$ are constants determined in iteration which is carried out in the following manner [13]:

1. First of all, we fix the coordinate radii of stellar surfaces at equator and pole. Also, we fix the value of $\rho_{b}$ at $r=0$.

2. We give a trial density configuration for $\rho_{b}$.

3. We solve field equations (C1)-(C4).

4. Since we have constraints imposed at procedure 1, we can determine $C, K$, and $\Omega_{s}$ using Eq. (C5).

5. Using Eq. (C5) with $C, K$, and $\Omega_{s}$ determined at procedure 4 , we calculate a new trial configuration for $\rho_{b}$.

6. Return to 2 , and repeat procedures $2-5$ until a sufficient convergence is achived.

Numerical computation is typically performed taking $\left(N_{r}, N_{\theta}\right)=(800,80)$ grid points, where $N_{r}$ and $N_{\theta}$ denote grid points for $0<r \leq r_{\max }$ and $0<\theta<\pi / 2$. We use homogeneous grids in $r$ and $y=\cos \theta$. We check our numerical code by comparing our numerical results for non-dimensional quantities $M K^{-n / 2}, J K^{-n}, R K^{-n / 2}$, and $\Omega_{s} K^{n / 2}$ with another results presented previously in ref. [16] for the case $n=1$. We find that our numerical results agree well with those in ref. [16] (inconsistency is less than 1\%). 
[1] M. van der Kils, astro-ph/9704272 and 9701006. See also references cited therein.

[2] M. C. Miller, F. K. Lamb, and G. B. Cook, astro$\mathrm{ph} / 9805007$.

[3] W. Kluzniak and R. V. Wagoner, Astrophys. J. 297, 548 (1985).

[4] S. W. Hawking and G. F. R. Ellis, The large scale structure of space-time(Cambridge University Press, 1973). See also references cited therein.

[5] R. O. Hansen, J. Math. Phys. 15, 46 (1974).

[6] W. G. Laarakkers and E. Poisson, gr-qc/9709033.

[7] For example, R. M. Wald, General Relativity (The University of Chicago Press, 1984), Chapter 7.

[8] F. J. Ernst, Phys. Rev. 167, 1175 (1968).

[9] G. Fodor, C. Hoenselaers, and Z. Perjes, J. Math. Phys. 30, 2252 (1989).

[10] F. D. Ryan, Phys. Rev. D 52, 5707 (1995).

[11] Algebraic manipulations were performed with the aid of Mathematica (S. Wolfram, Mathematica, Wolfram Research Inc., 1988).

[12] For example, S. L. Shapiro and S. A. Teukolsky, Black Holes, White Dwarfs, and Neutron Stars (Wiley, New York, 1983), chapter 12.

[13] H. Komatsu, Y. Eriguchi, and I. Hachisu, Mon. Not. R. astro. Soc. 237, 355 (1989).

[14] For example, A. P. Lightman, W. H. Press, R. H. Price and S. A Teukolsky, Problem Book in Relativity and Gravitation (Princeton Unversity Press, 1975), problem 16.17 .

[15] E. M. Butterworth and J. R. Ipser, Astrophys. J. 204, 200 (1976).

[16] G. B. Cook, S. L. Shapiro, and S. A. Teukolsky, Astrophys. J. 422, 227 (1994).

[17] J. F. Friedman, J. R. Ipser, and R. D. Sorkin, Astrophys. J. 325, 722 (1988). See also G. B. Cook, S. L. Shapiro, and S. A. Teukolsky, Astrophys. J. 398, 203 (1992). 
Table I. Numerical data for $n=1$ polytrope which we adopt in Sec. III. Note that $R_{e}$ denotes the circumferential radius at the stellar surface on the equatorial plane. Because $M \Omega_{s}(\equiv \chi)$ is an invariant quantity irrespective of scaling of $K$, we can calculate the spin angular frequency of stars from the following data approximately as $370 \mathrm{~Hz}(\chi / 0.016)\left(1.4 M_{\odot} / M\right)$.

\begin{tabular}{|c|c|c|c|c|c|c|c|c|}
\hline$\rho_{b, 0} K^{n}$ & $M K^{-n / 2}$ & $R_{e} K^{-n / 2}$ & $\Omega_{s} K^{n / 2}$ & $q$ & $Q_{2}$ & $q_{3}$ & $R_{\mathrm{ISCO}} / 6 M$ & $\Omega_{\mathrm{ISCO}} 6 \sqrt{6} M$ \\
\hline .200 & .158 & .870 & .061 & $9.48 \mathrm{E}-02$ & $3.53 \mathrm{E}-02$ & $6.9 \mathrm{E}-03$ & $\begin{array}{l}.953 \\
\end{array}$ & \begin{tabular}{|l|}
1.068 \\
\end{tabular} \\
\hline .217 & .160 & .852 & .064 & $9.43 \mathrm{E}-02$ & $3.19 \mathrm{E}-02$ & $6.1 \mathrm{E}-03$ & .953 & 1.069 \\
\hline .236 & .162 & .833 & .067 & $9.38 \mathrm{E}-02$ & $2.91 \mathrm{E}-02$ & $5.4 \mathrm{E}-03$ & .953 & 1.069 \\
\hline .257 & .163 & .814 & .070 & $9.34 \mathrm{E}-02$ & $2.66 \mathrm{E}-02$ & $4.8 \mathrm{E}-03$ & .952 & 1.070 \\
\hline .280 & .164 & .795 & .073 & $9.31 \mathrm{E}-02$ & $2.41 \mathrm{E}-02$ & $4.4 \mathrm{E}-03$ & .952 & 1.070 \\
\hline .305 & .164 & .776 & .076 & $9.28 \mathrm{E}-02$ & $2.22 \mathrm{E}-02$ & $4.0 \mathrm{E}-03$ & .952 & 1.070 \\
\hline .206 & .160 & .869 & .098 & $1.50 \mathrm{E}-01$ & $8.45 \mathrm{E}-02$ & $2.6 \mathrm{E}-02$ & .931 & 1.105 \\
\hline .224 & .162 & .851 & .103 & $1.49 \mathrm{E}-01$ & $7.66 \mathrm{E}-02$ & $2.3 \mathrm{E}-02$ & .930 & 1.106 \\
\hline .243 & .163 & .832 & .107 & $1.49 \mathrm{E}-01$ & $6.99 \mathrm{E}-02$ & $2.1 \mathrm{E}-02$ & .929 & 1.108 \\
\hline .265 & .164 & .813 & .112 & $1.48 \mathrm{E}-01$ & $6.36 \mathrm{E}-02$ & $1.8 \mathrm{E}-02$ & .928 & 1.109 \\
\hline .289 & .165 & .794 & .116 & $1.47 \mathrm{E}-01$ & $5.82 \mathrm{E}-02$ & $1.7 \mathrm{E}-02$ & .927 & 1.110 \\
\hline .314 & .165 & .775 & .121 & $1.47 \mathrm{E}-01$ & $5.36 \mathrm{E}-02$ & $1.5 \mathrm{E}-02$ & .927 & 1.111 \\
\hline .222 & .163 & .863 & .144 & $2.12 \mathrm{E}-01$ & $1.53 \mathrm{E}-01$ & $6.6 \mathrm{E}-02$ & .908 & 1.145 \\
\hline .241 & .165 & .844 & .150 & $2.11 \mathrm{E}-01$ & $1.39 \mathrm{E}-01$ & $5.9 \mathrm{E}-02$ & .906 & 1.149 \\
\hline .262 & .166 & .825 & .156 & $2.10 \mathrm{E}-01$ & $1.27 \mathrm{E}-01$ & $5.3 \mathrm{E}-02$ & .904 & 1.152 \\
\hline .285 & .166 & .806 & .163 & $2.10 \mathrm{E}-01$ & $1.17 \mathrm{E}-01$ & $4.8 \mathrm{E}-02$ & .902 & 1.154 \\
\hline .310 & .167 & .787 & .170 & $2.09 \mathrm{E}-01$ & $1.07 \mathrm{E}-01$ & $4.3 \mathrm{E}-02$ & .901 & 1.157 \\
\hline .237 & .166 & .859 & .181 & $2.60 \mathrm{E}-01$ & $2.11 \mathrm{E}-01$ & $1.1 \mathrm{E}-01$ & .891 & 1.176 \\
\hline .257 & .167 & .840 & .189 & $2.59 \mathrm{E}-01$ & $1.93 \mathrm{E}-01$ & $9.9 \mathrm{E}-02$ & .888 & 1.181 \\
\hline .280 & .168 & .820 & .197 & $2.58 \mathrm{E}-01$ & $1.77 \mathrm{E}-01$ & $8.9 \mathrm{E}-02$ & .885 & 1.186 \\
\hline .304 & .168 & .801 & .205 & $2.57 \mathrm{E}-01$ & $1.63 \mathrm{E}-01$ & $8.1 \mathrm{E}-02$ & .883 & 1.190 \\
\hline .231 & .167 & .876 & .206 & $3.03 \mathrm{E}-01$ & $2.86 \mathrm{E}-01$ & $1.7 \mathrm{E}-01$ & .882 & 1.196 \\
\hline .251 & .168 & .857 & .214 & $3.01 \mathrm{E}-01$ & $2.61 \mathrm{E}-01$ & $1.6 \mathrm{E}-01$ & .877 & 1.203 \\
\hline .272 & .169 & .838 & .223 & $3.00 \mathrm{E}-01$ & $2.40 \mathrm{E}-01$ & $1.4 \mathrm{E}-01$ & .873 & 1.210 \\
\hline .295 & .170 & .818 & .232 & $2.99 \mathrm{E}-01$ & $2.21 \mathrm{E}-01$ & $1.3 \mathrm{E}-01$ & .870 & 1.216 \\
\hline .249 & .172 & .883 & .258 & $3.71 \mathrm{E}-01$ & $3.84 \mathrm{E}-01$ & $2.9 \mathrm{E}-01$ & .862 & 1.237 \\
\hline .270 & .173 & .863 & .269 & $3.70 \mathrm{E}-01$ & $3.54 \mathrm{E}-01$ & $2.6 \mathrm{E}-01$ & .856 & 1.247 \\
\hline .292 & .173 & .844 & .279 & $3.68 \mathrm{E}-01$ & $3.27 \mathrm{E}-01$ & $2.4 \mathrm{E}-01$ & .851 & 1.257 \\
\hline .274 & .176 & .887 & .307 & $4.28 \mathrm{E}-01$ & $4.53 \mathrm{E}-01$ & $3.9 \mathrm{E}-01$ & .844 & 1.276 \\
\hline .294 & .177 & .869 & .318 & $4.26 \mathrm{E}-01$ & $4.22 \mathrm{E}-01$ & $3.5 \mathrm{E}-01$ & .837 & 1.288 \\
\hline
\end{tabular}




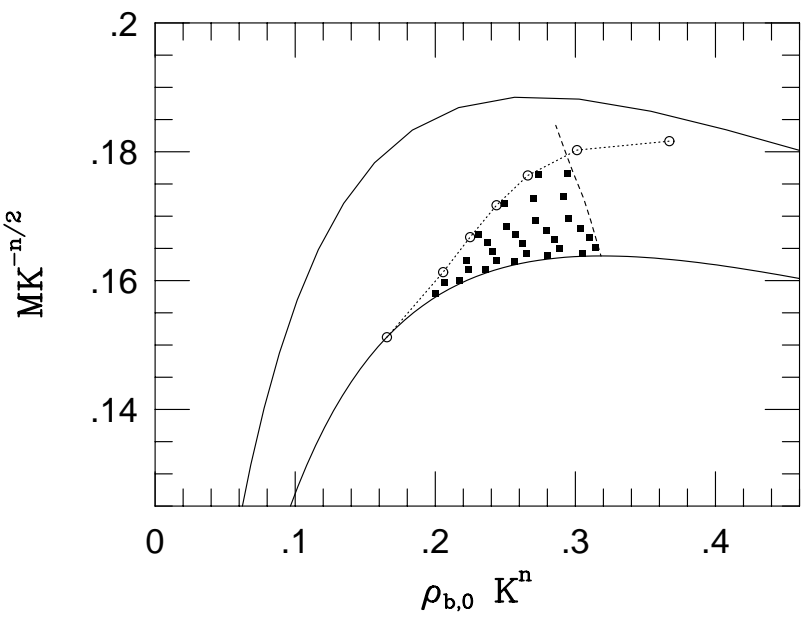

FIG. 1. Relation between mass $\left(M K^{-n / 2}\right)$ and density at $r=0\left(\rho_{b, 0} K^{n}\right)$ for $\mathrm{n}=1$ polytrope. Lower and upper solid lines denote the relations for the spherical star and for the rotating star at the mass shed limit. Dotted line (with open circles) divides two regions where the ISCO exists or not: It exists below the line. Dashed line divides the stable and unstable branches: The left-hand side is the stable region. Filled squares denote data sets which are compared to our analytic formulas.

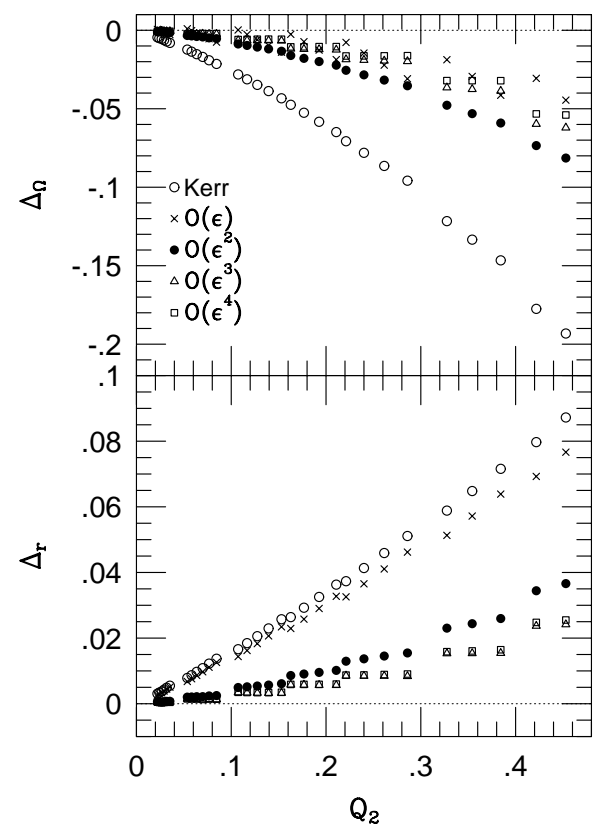

FIG. 2. $\Delta_{\Omega}$ and $\Delta_{r}$ as functions of $Q_{2}$ for $n=1$ polytrope. Open circles, crosses, filled circles, open triangles and open squares denote $\Delta_{a}^{\text {Kerr }}, \Delta_{a}(1), \Delta_{a}(2), \Delta_{a}(3)$ and $\Delta_{a}(4)(a=\Omega$ or $r)$, respectively. 